\title{
Smart Contact Tracing and Classifier System for
} Covid-19 Cases

\author{
Anasuodei Moko 1 , Amannah Constance Izuchukwu ${ }^{2}$ \\ ${ }^{1,2}$ Department of Computer Science and Informatics, Federal University Otuoke, Nigeria, \\ mokoaa@futuoke.edu.ng \\ ${ }^{2}$ Department of Computer Science, Ignatius Ajuru University of Education, Nigeria, aftermymsc@yahoo.com
}

Received Date : August 03, 2021 Accepted Date : August 22, 2021 Published Date : September 07, 2021

\begin{abstract}
The growing shreds of evidence and spread of COVID-19 in recent times have shown that to effortlessly and optimally tackle the rate at which COVID-19 infected individuals affect uninfected individuals has become a pressing challenge. This demands the need for a smart contact tracing method for COVID-19 contact tracing. This paper reviewed and analysed the available contact tracing models, contact tracing applications used by 36 countries, and their underlined classifier systems and techniques being used for COVID-19 contact tracing, machine learning classifier methods and ways in which these classifiers are evaluated. The incremental method was adopted because it results in a step-by-step rule set that continually changes. Three categories of learning classifier systems were also studied and recommended the Smartphone Mobile Bluetooth (BLE) and Michigan learning classifier system because it offers a short-range communication that is available regardless of the operating system and classifies based on set rules quickly and faster.
\end{abstract}

Key words: Covid-19, Classifier Systems, Smart Contact Tracing, Mobile Bluetooth.

\section{INTRODUCTION}

Contact tracing is of uttermost importance when there is a need to curb and contain the blow-out of communicable diseases. This form of tracing can be achieved both manually and digitally. Manual tracing before the introduction of the digital contact tracing over time has proven not to be fast enough, it is limited in small scale, interviews are performed in standard forms to verify the contacts up to 14 days after indications, which depends on contact ability to identify, remember and put down the names and mobile numbers of individuals that have in any form been in contact with them which has proved to be less effective. Digital Contact Tracing became highly recommended and adopted due to its large-scale coverage and effectiveness for the tackling and tracing of affected contacts. The number of mounting shreds of proof and spread has shown that to smoothly handle and manage the fast-increasing rate of Covid-19 is in the form of tracing contacts that have been in contact with infected individuals. (Cubanas et al, 2020). Developing innovative methods and tools has been paramount to aid health practitioners to keep track of contacts on a large scale. A more recent stochastic model has shown that if done in a short time, contact tracing can be helpful before an outbreak or COVID-19, which includes the detection, separation, confirmation, and treatment of contacts. (Garg et al, 2020).

The process of contact tracing is shown in Fig 1, in white paint in the dark shade, a method of tracing defined by public health as an identification process of individuals, who might have been or encountered an infected individual and compilation of more information about the contact. Even with the availability of different vaccines, contact tracing shows to be a more effective method for the prevention of infectious diseases, whereby cases and quarantines, social (physical) dissociation and disinfection measures such as handwashing with soap and water are adopted. (Asabere et al 2020). To prevent further spread, the contact tracing method is used to detect, and quarantine individuals exposed to an infectious person lately with COVID-19. This approach will crack the latest novel coronavirus transfer chain if used extensively and repress the epidemic by offering a greater opportunity for proper monitoring and contact tracing of would-be patients of the virus, who may be pre-symptomatic or symptomless based on the individual and as such if there is not an effective contact tracing method in place there is a high tendency of a headlong catastrophic spread.

In this regard, countries with an increasing number of infected persons are developing a process for digital contact tracking for mobile applications, using various innovations. (Lalmuanawma et al, 2020)

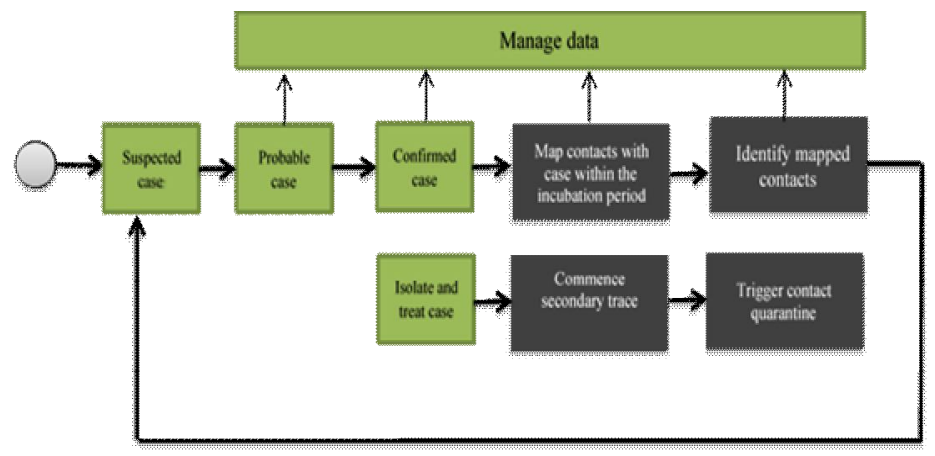

Figure 1: Identification of the disease case and tracing of contact (Garg et al, 2020) 
Contact tracing for covid-19 has faced difficulties in the time it takes to trace or identify a contact. The cluster of individuals who may have encountered the disease can spread to another group before they are apprised of an infected person. Infected persons do not have an idea of how they got infected, some infections are contacted from social gatherings and tracing the carrier is difficult. For fear of stigmatization infected persons play down on being exposed and due to the timing of the introduction of digital tools for contact tracing. Smart contact tracing solutions are necessary for locations or user proximity which can be performed in different ways. The automatic trace location or proximity of users can be used by placement or navigation devices such as GPS, Bluetooth, mobile networks, and thermal cameras.

The work is aimed at reviewing and analyzing available contact tracing models and their classifier system. The specific objectives are to review and analyze smart COVID-19 contact tracing models, recommend the most efficient smart contact tracing for COVID-19, review and analyze the contact tracing classifier system.

This study is limited to contact tracing of persons exposed to COVID-19 infected patients. Contact tracing of other infections, causing diseases are not reviewed and analyzed in this study. Consequently, the classifier system is also limited to the COVID-19 cases.

Smart contact tracing for COVID-19 will enable the identification and proper classification of individuals that have come in contact with COVID-19 via an infected individual or an infected object wherever they are located. Specifically, the study offers the following benefits: Preventing contact with positive cases which is essential for every effort to trace contacts. Proffer social support for individual compliance and medical supervision. protect uninfected individuals from future potential infections. Support private and public health practitioners with medical observation and approaches to strengthen case inquiry and contact tracing, as well as greatly aid in scaling up and reducing transmission. Decrease the wave of an epidemic or pandemic.

\section{REVIEW OF RELATED WORKS}

$\mathrm{Ng}$ et al., (2020) proposed a smart system for contact tracing (SCT) with the help of smartphones Bluetooth Low Energy (BLE) signals and a machine learning classifier which helped in obtaining a contact profile accurately and efficiently. The SCT system uses the services of the BLE feature that is non-connectable to create a signature packet, which gives an alert while the user is in an open space. The paper also highlighted two contributions of SCT, which are: classifying a user contact as elevated or minimal threat applying detailed juxtaposition sensing and hiding user profile by utilizing a communication protocol that preserves privacy. The work demonstrated that a BLE-based contact tracing system is a noteworthy infectious disease management and prevention remedy, and the SCT system provides significant results of using Received Signal Strength detecting closeness values between two people.

Martin et al., (2020) in an article review highlighted its achievement in threefold. Firstly, it used a concise but comprehensive assessment and categorization of the previously thorough frameworks proposed to realize contact tracing Second, it describes and categorizes the contact tracing application that European countries have previously implemented. Finally, it provides a non-precise rival model that does not only blends the applicable literature but also provides a new point of view on evaluating such systems but from a standpoint of security and data safety. Anyone who wants to improve understanding the various issues of this swiftly evolving and concise area can use the current work as a reference. It is also expected to trigger and nurture research efforts aimed at developing solutions that are worth focusing on technologically and in the aspects of data protection.

Garg et al., (2020) categorized the current ways of contact tracing; mobile network operators, call in detail, citizen application, mobile service provider application, and IoT based, then designed a novel privacy anonymous model. The study proposed a Radio Frequency Identification (RFID) Model proof of concept used, the suggested framework allows objects to send out and receive alerts when around a probable, labelled, or verified disease case or marked place or object as shown in Fig 1. Simulation results indicate that smart contracts can be deployed and called in less than one second, but in practice, they will be able to take close to 25 seconds on the Ethereum public blockchain. The findings likewise suggest that a minimum of $\$ 1.95$ is used to utilize the designed sample smart contracts and $\$ 0.34$ to invoke their functions.

Roy et al., (2020) introduced an IoT-based real-time solution that is new for contact tracing and checking the spread of COVID-19 infection. The introduced novel IoT device for identifying persons with COVID-19 possibly infected is what makes this approach unique. The potential of this basis is to substantively combine and affirm information concerning actual symptoms gotten from IoT tools on COVID-19 cases in other to offer a quick and easy method in monitoring the blowout, which is ultimately useful for the effective utilization of obtainable test kits for COVID-19. The study simulation results validate the effectiveness of tracing methods for transmissible diseases such as COVID-19.

Nasarjpour et al., (2020) examined the need for technologies based on IoT for COVID-19. The IoT-related technologies and their structures were observed in three stages, which are "Early Diagnosis," "Quarantine Time," and "After Recovery." In combating COVID-19, each phase was examined using the role of IoT-enabled and different connected technologies like wearables and smartphone applications etc. The study 
believes that IoT technology could be remarkably useful in combating COVID-19 and key attention needs to be given to data privacy, by securely employing appropriate IoT technology, more patients can engage in their treatment utilizing IoT devices with satisfaction. Authorities and healthcare workers will be capable of responding to pandemics more effectively using this method, as a result, the impact of such diseases can be significantly reduced.

Urbanowicz and Moore (2009) aimed at providing a simple starting point for researchers of diverse backgrounds taking an interest in choosing or creating their LCS. A basic yet detailed overview is included, as well as a historic account and a roadmap of algorithmic elements highlighting variations in substitute LCS operations.

$\mathrm{Hu}$ (2020) proposed a new IoT-based architecture that provides a quick fix for contact tracing and general disease confinement potency based on conventional epidemiological models and simulation results. The study provides a foundation for sustainable blueprints and evaluations of IoT-based contact tracing technologies, as well as to support data-driven collaborative measures to counter modern and imminent infectious diseases. The findings show that, even when there is a fierce lockdown, an IoT-Contact Tracing System can still play an important position in the initial restraint of disease spread.

Banerjee, et al., (2020) proposed a 'ProxiTrak', which is an accurate and powerful efficient solution that uses GPS (Global Positioning System) and BLE data to form graph-based contact patterns, resulting in a well-informed decision and insightful visualization capabilities.

Asabere et al., (2020) proposed a socially aware recommendation algorithm of individuals who are most likely infected with COVID-19 (SARPPIC), using closeness uniqueness to calculate the occurrence of specific contact points in a social network that has had a connection with an infected contact point (COVID-19 patient). When evaluated by comparing COV-1 and COV-2 (when delta equates to 0.1 ) on the dataset from HEXACO-60-ATU, the results showed. SARPPIC surpasses COV-1 and COV-2 based on four evaluation metrics. SARPPIC greatly improves (approximately $10 \%$ in precision, $16 \%$ in recall, $49 \%$ in $\mathrm{F} 1$ and $13 \%$ in $\mathrm{AM}$ when $\mathrm{N}$ is 11. Moreover, this suggests that the modified outline is a combination of high Betweenness Centrality (BC) used to calculate the significances of the social graph network contact points, and its tie power can aid in the generation of more accurate social recommendations relating to likely COVID-19 infected individuals.

Chen and See (2020) studied artificial intelligence (AI) being employed at several levels of the health care system to address the global health crisis. The study highlighted that AI, on the other hand, also has prospective benefits and drawbacks. Results show that in the 11 papers selected for review in the study, that COVID-19 was treated with artificial intelligence in the tetrad, which is: diagnosis, public health, clinical decision making, and therapeutics. With so many issues encountered and the constant upsurge in the figure of COVID-19 cases, AI has the potential to increase human efforts considerably, which would otherwise be overloaded by high numbers of affected roles.

Cabañas et al (2021) in the frame of reference to the COVID-19 pandemic, opined that the currently deployed contact-tracing mobile applications have managed to fail to provide a more effective approach. The work offers a solution that makes use of the already obtainable geolocation that have very higher absorption levels in nearly all countries that use mobile application for contact tracing. The study proposed a different contact-tracing resolution that applies geolocation information of numerous users to classify and pinpoint individuals who in any form has been in contact with an individual that has been confirmed positive tagging them as risk contacts.

\subsection{Application used by Countries for Contact Tracing}

Diverse countries have applied the notion of Machine Learning and Artificial Intelligence to facilitate contact tracing. Lalmuanawma et al., (2020) study shows, over 36 countries have significantly used digital contact tracing to reduce and enhance the quality of old-fashioned diagnosis procedures regarding centralized, decentralized or the use of both techniques. The location tracking method employed by the various countries is predominantly the Bluetooth and GSM tracking method. 11 countries from the 36 adopted the Bluetooth location tracking application, 11 countries combined the Bluetooth with the Google/Apple location tracking application, 4 countries combined the Bluetooth and GSM tracking application, another 4 used the GPS and 2 countries combine the GPS and GSM location tracking method. China which recorded one the highest in COVID-19 cases combined 3 location tracking techniques which are the GPS, GSM and the credit card transaction history, South Korea used the mobile device tracking data and card transaction data and New Zealand adopted the contact details and physical address to achieve digital contact tracing and finally, India used the location-generated social graph.

\section{METHODOLOGY}

The incremental method adopted for this case provides training instances at a time, which results in a rule set that continually changes by inserting each additional rule while using the learning classifier system. The system solution will continuously change about a continuous input stream since this form of learning does not have a present endpoint.

The need for sensing cannot be ruled out when looking at building a smart system, even if COVID-19 remains under, the spread from individual to person via breathing droplet and 
from polluted surfaces and substances, collectively known as 'things' of different viability on different surfaces. Therefore, contact monitoring must target individuals and objects, such that transmissions from individual to object and object to person can be tracked, to show peer-to-peer communications among a Susceptible (S) or Infectious (I) person and a Recovered (R) individual. Tracking human-to-object is to trace an individual in S/I states who can pollute objects. Any fit person who may be tainted by the tainted object is the object-to-person. Contact tracing, for example, where a person enters a building with detecting capacity in the premises for personal or object-to-person. Sensing technologies are required to detect contact to contagious diseases through individual and object-to-person transmissions. The sensing technology employed by this work is a smartphone application.

\subsection{Smart Contact Tracing System Components}

\section{A. Mobile Bluetooth Technology}

Bluetooth Low Energy (BLE) technology offers communication over a short distance around the $2,4 \mathrm{GHz}$ ISM band. BLE technology provides a short range of communication. It is pervasive and has several clever gadgets such as smartwatches, earphones, and so on. As the primary messaging platform, smart thermostats, etc. Besides, BLE is readily available regardless of the operating system on most modern smartphones. There are two separate ways of contact with BLE: non-connectable publicity and connectable publicity, (Ng. et al 2020).

\section{B. Classifier System}

Classifier systems are designed to use genetic algorithms to research conditions and action learning, rules-based systems, they can provide a solution when faced with complex problems, these rules-based, multifaceted, algorithms of machine-learning have been developed into artificial and evolutionary biology. The Learning Classifier System (LCS) concept inspired several implementations that were adapted to manage various problem areas (e.g., independent robot, classification, knowledge discovery, also modelling), an area that is becoming more noticeable and needed. (Holland, 1999).

LCS is epidemiology, which demands more and more powerful instruments to promote the discovery of etiology.

(Ryan and Jason, 2009).

A classifier system includes:

i. Production Rules, also known as classifiers

ii. Working memory

iii. Input Sensors or decoders

iv. Output or effectors

v.

\subsection{Paradigm of Learning Classifier System}

The Pittsburgh Approach: This is the most like the standard concept of the Genetic Algorithm. Where each person is a full answer to the classification problem. Conventionally, this means that everyone is a set of rules with varying lengths. A well-known example of this approach is the Genetic Algorithm Batch-Incremental Concept Learner (GABIL).

The Michigan Approach: Every single person (classifier) is a single rule, and the entire population works together to help address the classification problem. Rules will be assessed by example, and after sufficient trials and obtaining a positive or negative benefit, rule fitness will be progressively modified with this reward, and good rules will have increased fitness.

The Iterative Rule Learning Approach: Individuals end up competing in the same way as in a standard GA; a single GA run produces one rule, which is then run iteratively to learn all rules that fix the problem. Instances that have already been covered by previous rules are erased from the next iteration's training set, also known as separate-and-conquer Initial Training. (Urbanowicz and Moore, 2019).

\subsection{Algorithm for Learning Classifier Architecture}

Step 1:

Get Tracing instance from the environment

Step 2:

Find all rules in the population that come up with a condition identical to the attribute values of the instance

Step 3:

Match parameters by comparing all rules

Step 4:

IF all specify attributes match move rule to Match Set

(M), IF not Discard

Step 5:

Divide Match Set(M) to correct set (C) and incorrect set (I)

Step 6:

Move rules that have a class prediction that match instance of the training set to a correct set (C) and those that do not to incorrect set (I)

Step 7:

If no rule makes it to the correct set (C) apply the covering mechanism

Step 8:

Update parameters of the classifier

Step 9:

Subsume by covering all problem space.

Step 10:

Output 


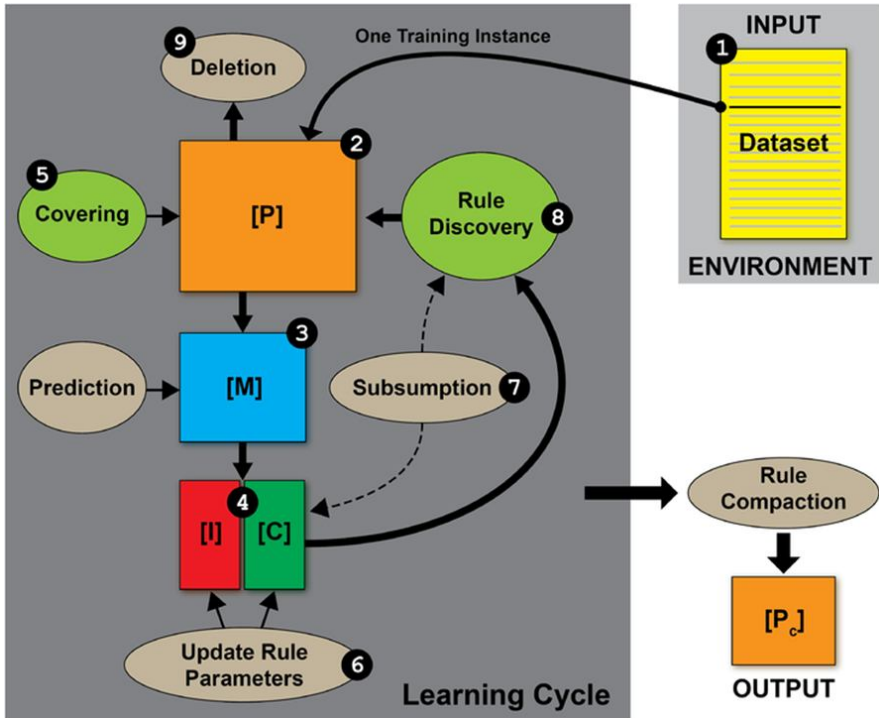

Figure 2: Michigan-Style Learning Classifier System (Source: Bacardit \& Butz, 2007)

\subsection{Machine Learning Classifier}

Classification a supervised machine learning method that is performed on structured or unstructured data is when point sets of a class are predicted, which are often referred to as categories or targets/labels. The classifier on the other hand is an algorithm mapping used for input data for a particular classification. Different classifiers that are used for classification are:

Logistic Regression which illustrates the probable results of a particular test demonstrated using a logistic function; Naive Bayes algorithm which is founded on Bayes's theorem assuming that each set of features is independent; Stochastic Gradient Descent is a tactic used to fitting linear models, quite convenient especially if there is a very vast number of iterations; K-Nearest Neighbour is a procedure of slothful knowledge because it does not try to build an inclusive internal model, but basically stores training data instances; Decision Tree are set of rules that is used to categorize data, given the data features along with their classes; Random Forest is a meta-estimator for several sub-samples of the dataset that fits in several decision tree classes and utilizes an average to enhance prediction accuracy and overfitting.; Support Vector Machine uses training data to represent a concise gap in space such that feasible points in space are divided into categories, which uses new examples to map into the same area to predict which portion of the gap they drop. (Garg, 2018)

\subsection{Classifier Evaluation Method}

The most essential part after training is that the classifier is evaluated to check its pertinency, and it is evaluated by many accurate decisions made divided by a total number of test samples which gives the accuracy of the classifier used. The methods used for evaluation are Cross-Validation, Holdout,
Receiver Operating Characteristics (ROC curves), Precision and Recall.

Table 1: Contact Tracing Techniques, classifier system, and recommendations.

\begin{tabular}{|c|c|c|c|}
\hline $\mathrm{S} / \mathrm{N}$ & $\begin{array}{l}\text { Smart } \\
\text { COVID-19 } \\
\text { Contact Tracing } \\
\text { Technique }\end{array}$ & Classifier System & $\begin{array}{l}\text { Recommendation } \\
\mathrm{s}\end{array}$ \\
\hline 1. & $\begin{array}{l}\text { Smartphones } \\
\text { Bluetooth Low } \\
\text { Energy (BLE) } \\
\text { Signals. (Ng et } \\
\text { al 2020). }\end{array}$ & $\begin{array}{l}\text { Machine learning } \\
\text { classifier }\end{array}$ & $\begin{array}{l}\text { Variable } \\
\text { attenuation paths } \\
\text { caused by } \\
\text { smartphone } \\
\text { placement on } \\
\text { different body } \\
\text { positions can have } \\
\text { a significant } \\
\text { impact on } \\
\text { accuracy; } \\
\text { therefore, } \\
\text { research that } \\
\text { should affect the } \\
\text { attenuation routes } \\
\text { on both sides } \\
\text { needs to be } \\
\text { reviewed and an } \\
\text { adaptive route } \\
\text { loss system } \\
\text { proposed to adapt } \\
\text { to such diversity } \\
\text { in attenuation } \\
\text { routes. }\end{array}$ \\
\hline 2. & $\begin{array}{l}\text { Socially aware } \\
\text { recommendatio } \\
\text { n (SARPPIC). } \\
\text { (Asabere et al } \\
\text { 2020). }\end{array}$ & $\begin{array}{l}\text { Recommendation } \\
\text { Algorithm }\end{array}$ & $\begin{array}{l}\text { Social properties, } \\
\text { such as the } \\
\text { centrality of } \\
\text { proximity, social } \\
\text { neighbours and } \\
\text { the idea of } \\
\text { individuality, } \\
\text { must be } \\
\text { considered and } \\
\text { utilized in the } \\
\text { recent COVID-19 } \\
\text { epidemic. }\end{array}$ \\
\hline 3. & $\begin{array}{l}\text { Anonymity } \\
\text { Preserving } \\
\text { IoT-Based for } \\
\text { COVID-19 } \\
\text { (Garg et al } \\
\text { 2020) }\end{array}$ & $\begin{array}{l}\text { Passive RFID } \\
\text { transceiver }\end{array}$ & $\begin{array}{l}\text { RFID solutions } \\
\text { must be } \\
\text { implemented in } \\
\text { conjunction with } \\
\text { established } \\
\text { frontend } \\
\text { applications or the } \\
\text { latest frontend } \\
\text { applications. } \\
\text { Coming studies } \\
\text { should also try to } \\
\text { diminish the } \\
\text { amount when } \\
\text { scaling the } \\
\text { solution. }\end{array}$ \\
\hline 4. & $\begin{array}{l}\text { ProxiTrak: A } \\
\text { Smart Contact } \\
\text { Tracing System } \\
\text { to Fight }\end{array}$ & $\begin{array}{l}\text { K-nearest } \\
\text { Neighbour } \\
\text { classifier }\end{array}$ & $\begin{array}{l}\text { As an immediate } \\
\text { next step, } \\
\text { incorporate } \\
\text { improved privacy }\end{array}$ \\
\hline
\end{tabular}




\begin{tabular}{|l|l|l|l|}
\hline & $\begin{array}{l}\text { COVID-19 that } \\
\text { uses GPS } \\
\text { (Global } \\
\text { Positioning } \\
\text { System) and } \\
\text { BLE data. } \\
\text { (Banerjee et al } \\
2020)\end{array}$ & $\begin{array}{l}\text { management and } \\
\text { advanced } \\
\text { visualization. }\end{array}$ \\
\hline 5. & $\begin{array}{l}\text { Efficient } \\
\text { Monitoring and } \\
\text { Contact Tracing } \\
\text { for COVID-19: } \\
\text { A Smart } \\
\text { IoT-Based } \\
\text { Framework } \\
\text { (Roy et al 2020) }\end{array}$ & $\begin{array}{l}\text { D2D based } \\
\text { relay IoT-based } \\
\text { contact tracing } \\
\text { algorithm }\end{array}$ & $\begin{array}{l}\text { To create } \\
\text { prototypes and } \\
\text { then fine-tune } \\
\text { them into } \\
\text { large-scale } \\
\text { products that can } \\
\text { be used. }\end{array}$ \\
\hline
\end{tabular}

\section{DISCUSSION}

The emergence of COVID-19 has triggered global economic problems, leading to underemployment, financial sector declines, and unparalleled borrowing costs and lots more, of which if not contained will lead many more people to poverty. The Internet of Things (IoT) and artificial intelligence (AI) can support in resolving the critical questions posed by the COVID-19 contagion. The challenges involved in the effective method for COVD-19 contact tracing cannot be overemphasized, because constantly despite the breakthrough achieved with different drugs and vaccines, there is still a persistent boost in the sum of cases regular. To handle and tackle the method of smart COVID -19 contact tracing, effectively with the use of Bluetooth system on smartphones and the Michigan learning classifier system. This method of classifier learning system uses a rule-based method properly to classify individuals who have in any form encounter the virus via an infected individual or an object swiftly with negligible errors. A succinct study has been done on the various contact tracing methods being used by different countries and it is appropriate that whatever methods is being adopted the goal is to achieve a minimal slight error of which our paper seeks to address.

\section{CONCLUSION}

Contact tracing is one of several complementary strategies for COVID-19 infection reduction, stoppage, and reversal, there has been a major shift globally recently in the way people live and interact with their environment. Contact tracing remains the only competent action in extreme occurrence situations for household contacts, contacts from overcrowded or inaccessible surroundings so that cases and their contacts are detached from affecting other individuals. This paper examined smart contact tracing, technologies, and applications, the idea of introducing the smart technology is to help properly trace, predict, detected infected objects and isolate infected individuals from non-infected people. Current contact tracing measures were discovered to be ineffective in the presence of moving objects, prompting the development of a smart contact tracing system and classifier learning systems. The study reveals that a Bluetooth Low Energy (BLE)-based system for contact tracing is an extensively epidemic management and prevention method used.

\section{REFERENCES}

1. Asabere, N. Y., Acakpovi, A., Ofori, E. K., Torgby, W., Kuuboore, M., Lawson, G., \& Adjaloko, E. (2020, November 16). SARPPIC: Exploiting COVID-19 Contact Tracing Recommendation through Social Awareness. Computational and Mathematical Methods in Medicine; Volume 2020, 14pages Hindawi.

2. Banerjee, S., Chandel, V., \& Ghose, A. (2020). ProxiTrak: A Smart Contact Tracing System to Fight COVID-19.

3. Bacardit, J., \& Butz, M. V. (2007). Learning Classifier Systems (pp. 282-290). Springer Berlin Heidelberg.

4. Braithwaite, I., Callender, T., Bullock, M., \& Aldridge, R. W. (2020). Automated and partly automated contact tracing: A systematic review to inform the control of COVID-19. The Lancet Digital Health, 2(11), 15pages.

5. Cabañas, J. G., Cuevas, Á., Cuevas, R., \& Maier, M. (2021). Digital Contact Tracing: Large-scale Geolocation Data as an Alternative to Bluetooth-based Apps' Failure.

6. Garg, L., Chukwu, E., Nasser, N., Chakraborty, C., \& Garg, G. (2020). Anonymity Preserving IoT-Based COVID-19 and Other Infectious Disease Contact Tracing Model. IEEE Access, 8,

7. Garg, R. (2018, January 19). 7 Types of Classification Algorithms. Analytics India Magazine.https://analyticsindiamag.com/7-types-clas sification-algorithms/

8. Holland, J., Booker, L., Colombetti, M., Dorigo, M., Goldberg, D., Forrest, S., Riolo, R., Smith, R., Lanzi, P. L., Stolzmann, W., \& Wilson, S. (1999). What Is a Learning Classifier System? In Learning Classifier Systems: From Foundations to Applications (Vol. 1813, p. 32).

9. Hu, P. (2020). IoT-based Contact Tracing Systems for Infectious Diseases: Architecture and Analysis. Retrieved from: http://arxiv.org/abs/2009.01902 Accessed 4, February 2021

10. Lalmuanawma, S., Hussain, J., \& Chhakchhuak, L. (2020). Applications of machine learning and artificial intelligence for Covid-19 (SARS-CoV-2) 
pandemic: A review. Chaos, Solitons, and Fractals, 139,

11. Martin, T., Karopoulos, G., Hernández-Ramos, J. L., Kambourakis, G., \& Nai Fovino, I. (2020). Demystifying COVID-19 Digital Contact Tracing: A Survey on Frameworks and Mobile Apps. Wireless Communications and Mobile Computing, $1-29$.

12. Nasajpour, M., Pouriyeh, S., Parizi, R. M., Dorodchi, M., Valero, M., \& Arabnia, H. R. (2020). Internet of Things for Current COVID-19 and Future Pandemics: An Exploratory Study. Journal of Healthcare Informatics Research, 4, 40pg

13. P. C. Ng, P. Spachos and K. N. Plataniotis, "COVID-19 and Your Smartphone: BLE-Based Smart Contact Tracing," in IEEE Systems Journal, doi: 10.1109/JSYST.2021.3055675.

14. Roy, A., Kumbhar, F. H., Dhillon, H. S., Saxena, N., Shin, S. Y., \& Singh, S. (2020). Efficient Monitoring and Contact Tracing for COVID-19: A Smart IoT-Based Framework. IEEE Internet of Things Magazine, 3(3), 17-23. Accessed 3, February 2021

15. Stiepan, D. (n.d.). What is contact tracing, and why is it important in the fight against COVID-19? Retrieved from: https://newsnetwork.mayoclinic.org/discussion/whatis-contact-tracing-and-why-is-it-important-in-fight-a gainst-covid-19/, Accessed 4, February 2021.

16. Urbanowicz, R. J., \& Moore, J. H. (2009). Learning Classifier Systems: A Complete Introduction, Review, and Roadmap. Journal of Artificial Evolution and Applications, 2009, 25. 